\title{
Port Tanger Méditerranée : étude géologique et géotechnique, et analyse du risque de liquéfaction à partir des essais in situ
}

\author{
Mohammed Reda AOULAD MANSOUR ${ }^{1,3}$, Abdel-Ilah MIHRAJE ${ }^{2}$, \\ Jamal Eddine STITOU MESSARI ${ }^{3}$
}

1. Agence Spéciale Tanger Méditerranée (TMSA), ZFE de Ksar El Majaz, Oued Rmel Route de Fnideq, Maroc. aouladmansour@gmail.com

2. Direction de l'Aménagement du Port Tanger Méditerranée (DAPTM), ZFE de Ksar El Majaz Oued Rmel Route de Fnideq, Maroc.

amihraje@yahoo.fr

3. Université Abdelmalek Essaadi, Faculté des Sciences de Tétouan, BP 2121 Tétouan, Maroc.

jstitou@uae.ma

\section{Résumé :}

Le site du Port Tanger Méditerranée, situé sur les flyschs de Tisirène de l'unité de Tanger faisant partie du domaine externe rifain, a fait l'objet de plusieurs campagnes de reconnaissance géotechnique qui ont permis d'établir une synthèse géologique et géotechnique et de repérer des couches de sables saturés susceptibles de liquéfier sous l'effet des séismes. L'analyse du risque de liquéfaction est conduite sur la base des essais in situ (CPTU) et selon les deux séismes considérés dans le projet. Elle montre que la zone à risque est réduite et se situe entre les essais CPT22 et 25 et que le séisme lointain est plus néfaste pour le terrain que le séisme de source proche. La charge appliquée par l'ouvrage réduit ou atténue selon les cas le potentiel de liquéfaction, il subsiste cependant dans la partie superficielle du sol sur environ 3m de hauteur.

Soumis le 31 janvier 2012, accepté le 24 février 2014, en ligne le 31 mars 2014.

La seule version examinée est celle écrite en français. La ou les autres versions n'étant pas examinées par le comité de rédaction de la revue, sont donc publiées sous l'entière responsabilité du ou des auteurs.

A TRANSLATED VERSION IN ENGLISH IS AVAILABLE ONLINE

Pour citer cet article :

AOULAD MANSOUR M.R., MIHRAJE A.-I., STITOU MESSARI J.E. (2014). Port Tanger Méditerranée : étude géologique et géotechnique, et analyse du risque de liquéfaction à partir des essais in situ. Revue Paralia, Vol. 7, pp n01.1-n01.12.

DOI: http://dx.doi.org/10.5150/revue-paralia.2014.n01 


\section{Introduction}

Situé entre les villes de Ksar Sghir et Fnideq à l'extrémité nord occidentale du Maroc, le site du port Tanger Med est traversé par l'embouchure d'Oued Rmel. Les assises géologiques sont des alternances de grès et de pélites du domaine des flyschs rifains de la nappe de Tisirène, les bancs métriques gréseux dominent sur la rive droite du port, tandis que sur la rive gauche dominent les pélites. Ce travail consiste en :

- une synthèse géologique et géotechnique des données des différentes campagnes de reconnaissance réalisées dans la zone du port depuis le démarrage des études en 2002 ;

- une étude du risque de liquéfaction des sols sableux menée à partir des essais in situ.

\section{Etude géologique et géotechnique}

\subsection{Campagnes géotechniques et sondages réalisés}

Le site du port de Tanger Méditerranée a fait l'objet de plusieurs campagnes de reconnaissance géotechnique échelonnées dans le temps et conduites par plusieurs laboratoires (LPEE, FUGRO, SEACORE, GTEC) voir tableau 1. Ainsi plusieurs sondages et essais ont été réalisés, leur implantation est donnée sur la figure 1. L’ensemble des données est utilisé dans cette étude.

Tableau 1. Recensement des campagnes d'essais géotechniques réalisés au port Tanger Med.

\begin{tabular}{|c|c|c|}
\hline Campagne & Laboratoire et date & Types d'essais \\
\hline Reconnaissances préliminaires & LPEE, Maroc-2003 & Sondages carottés \\
\hline Reconnaissances du site du port & FUGRO, France - 2004 & Sondages carottés $+C P T^{*}$ \\
\hline $\begin{array}{l}\text { Reconnaissances des ouvrages } \\
\text { de protection }\end{array}$ & $\begin{array}{l}\text { SEACORE, Royaume-Uni- } \\
2005\end{array}$ & $\begin{array}{l}\text { Sondages carottés }+C P T \\
+S P T^{* *}\end{array}$ \\
\hline Terre-pleins & LPEE, Maroc - 2004 & $\begin{array}{l}\text { Sondages carottés }+C P T+ \\
\text { pressiomètres }\end{array}$ \\
\hline Quai à conteneur & LPEE, Maroc - 2005/2006 & $\begin{array}{l}\text { Sondages carottés + } \\
\text { pressiomètres }\end{array}$ \\
\hline Quai à conteneur & GTEC, Belgique -2005 & $C P T$ \\
\hline Terre-pleins complémentaires & LPEE, Maroc -2005 & $\begin{array}{l}\text { Sondages carottés }+C P T+ \\
\text { pressiomètres }\end{array}$ \\
\hline Terre-pleins (TC1) & FUGRO, France - 2006 & Sondages carottés + SPT \\
\hline
\end{tabular}

Nota : * CPT : Cone Penetration Test ; ** SPT : Standard Penetration Test. 


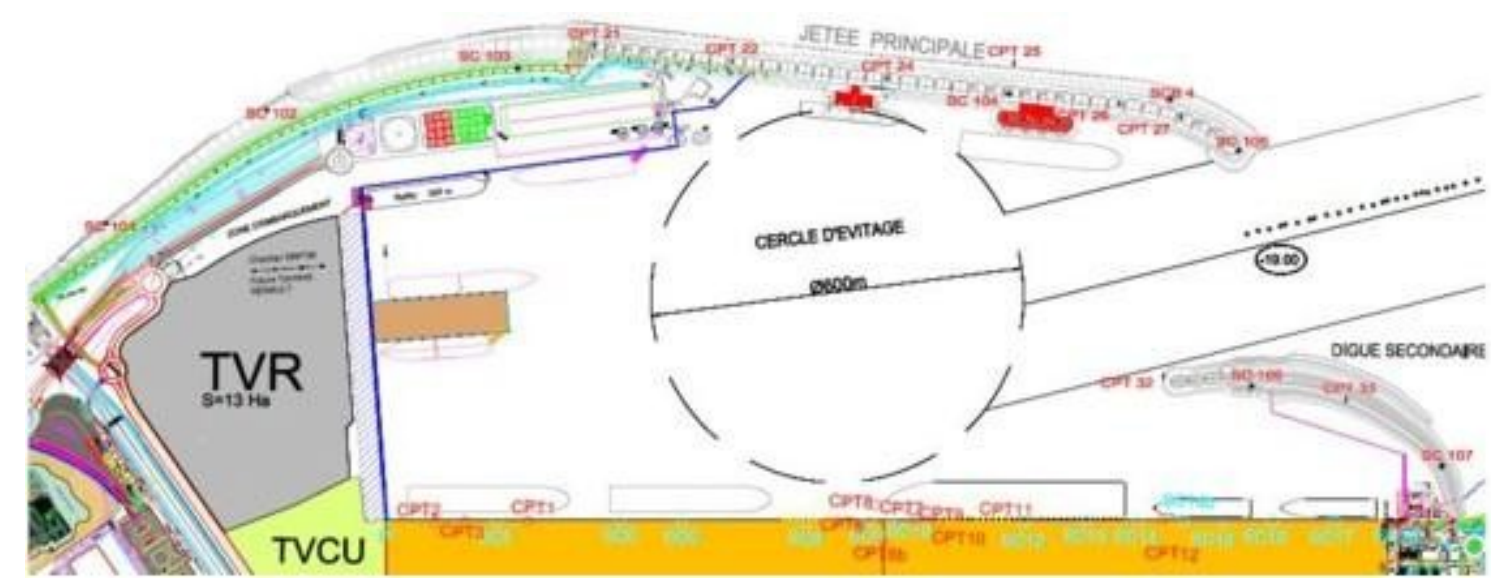

Figure 1. Plan de situation des sondages et essais.

\subsection{Réalisation de la carte du substratum}

Les données des différentes campagnes traitées par les logiciels SURFER et COVADIS ont permis d'établir une carte du substratum et le bloc diagramme correspondant (figure 2). Ces derniers montrent la présence de 3 vallées dont l'ampleur est variable et correspondent respectivement à Oued Rmel, Oued Chaâba et un petit cours d'eau.

Il est à signaler également que le substratum plonge brutalement vers le large. La superposition de la carte avec le plan de masse du port (figure 2) permet de localiser ces vallées et montre qu'elles traversent les terre-pleins, l'emplacement du quai à conteneurs et certaines se prolongent même sous l'emprise des ouvrages de protection.

\subsection{Réalisation des coupes géologiques}

Trois coupes géologiques ont été établies à l'emplacement des principaux ouvrages du port (figure 3), à savoir les ouvrages de protection et le quai à conteneur, en vue d'identifier le sol de fondation et de faire ressortir les principaux problèmes qu'il pourra générer.

Ces coupes montrent l'existence d'une épaisse couche de sables et de sables silteux en couleur jaune et orange sur la figure 4, à l'emprise des ouvrages de protection (AOULAD MANSOUR, 2007 ; AOULAD MANSOUR et al., 2011).

La coupe type montre du haut vers le bas :

- des sables propres en couches épaisses de $15 \mathrm{~m}$, ils sont composés en moyenne de $87 \%$ de sables, $12 \%$ de silts et $1 \%$ d'argiles ;

- des sables silteux argileux dont l'épaisseur atteint 6,3 m et composés de 39\% de sables, 33\% de silts et 28\% d'argiles, avec un indice de plasticité $I_{P}$ de 16,2\% (valeur moyenne sur la couche) ;

- des silts argileux, sous forme de lentilles minces, repérés uniquement au forage SCB4, ils sont formés de $34 \%$ de sables, $52 \%$ de silts et $14 \%$ d'argiles ; l'indice de plasticité $\mathrm{I}_{\mathrm{P}}$ de cette formation est de $10 \%$; 
- des graviers pour une épaisseur de 1 à $4 \mathrm{~m}$;

- des silts sableux composés de $40 \%$ de sables, 39\% de silts et $21 \%$ d'argiles ; l'indice de plasticité $I_{P}$ correspondant est de 14,3\% ;

- le substratum gréso-pélitique dont la partie superficielle est altérée.
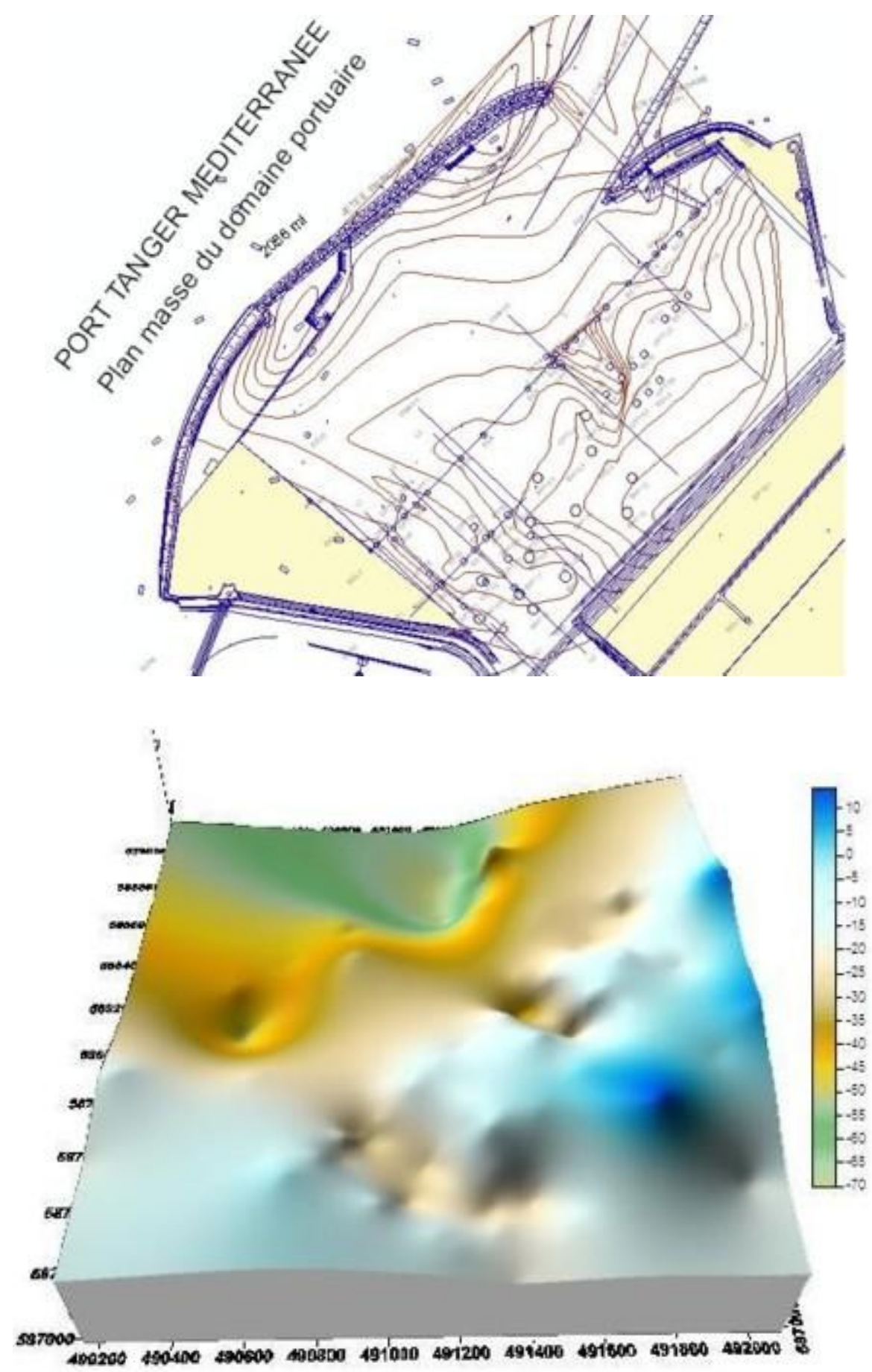

Figure 2. Plan du port en haut et en bas bloc diagramme du substratum (échelle montrant la profondeur du substrat par rapport au niveau marin). 


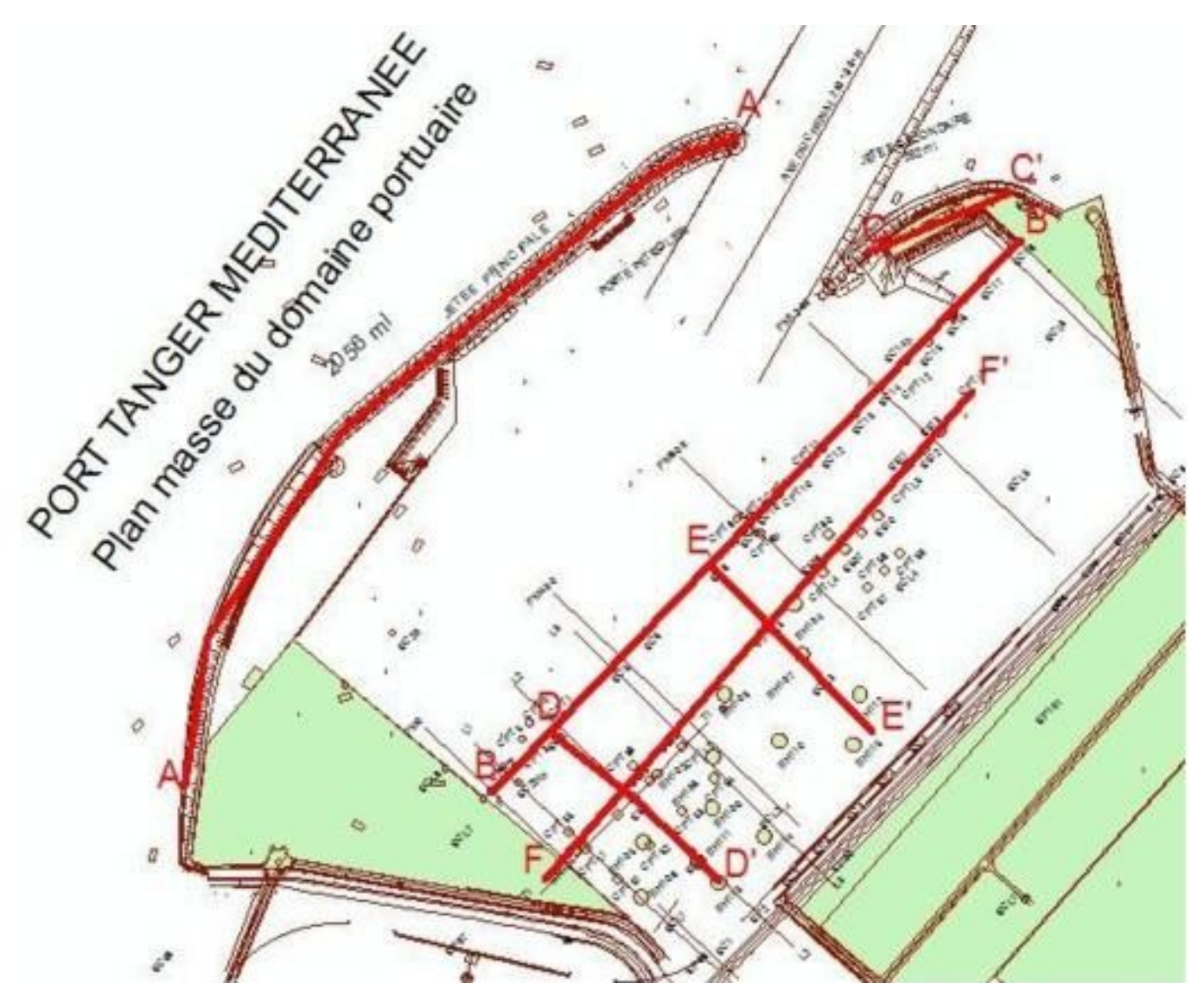

Figure 3. Localisation des essais et des coupes par rapport aux ouvrages du port.

Dans ces formations saturées d'eau, il y a un risque éventuel de liquéfaction sous l'effet des séismes. Il convient d'évaluer ce risque lors du projet.

Dans l'emprise du quai à conteneur, les vallées cumulent plus de 30m de matériaux dont des sables, sables silteux et de silts argileux impropres à la fondation du quai. Ces couches peuvent générer des tassements inacceptables (AOULAD MANSOUR, 2007 ; AOULAD MANSOUR et al., 2011). Ces matériaux impropres à la fondation du quai à conteneur construit avec des blocs préfabriqués en béton, ont été dragués jusqu'au substratum. Ils ont été substitués par des matériaux granulaires de granulométrie adaptée en provenance de carrière et vibro-compactés pour en améliorer la densification et minimiser le risque de tassement sous le quai à conteneur (figure 4). 

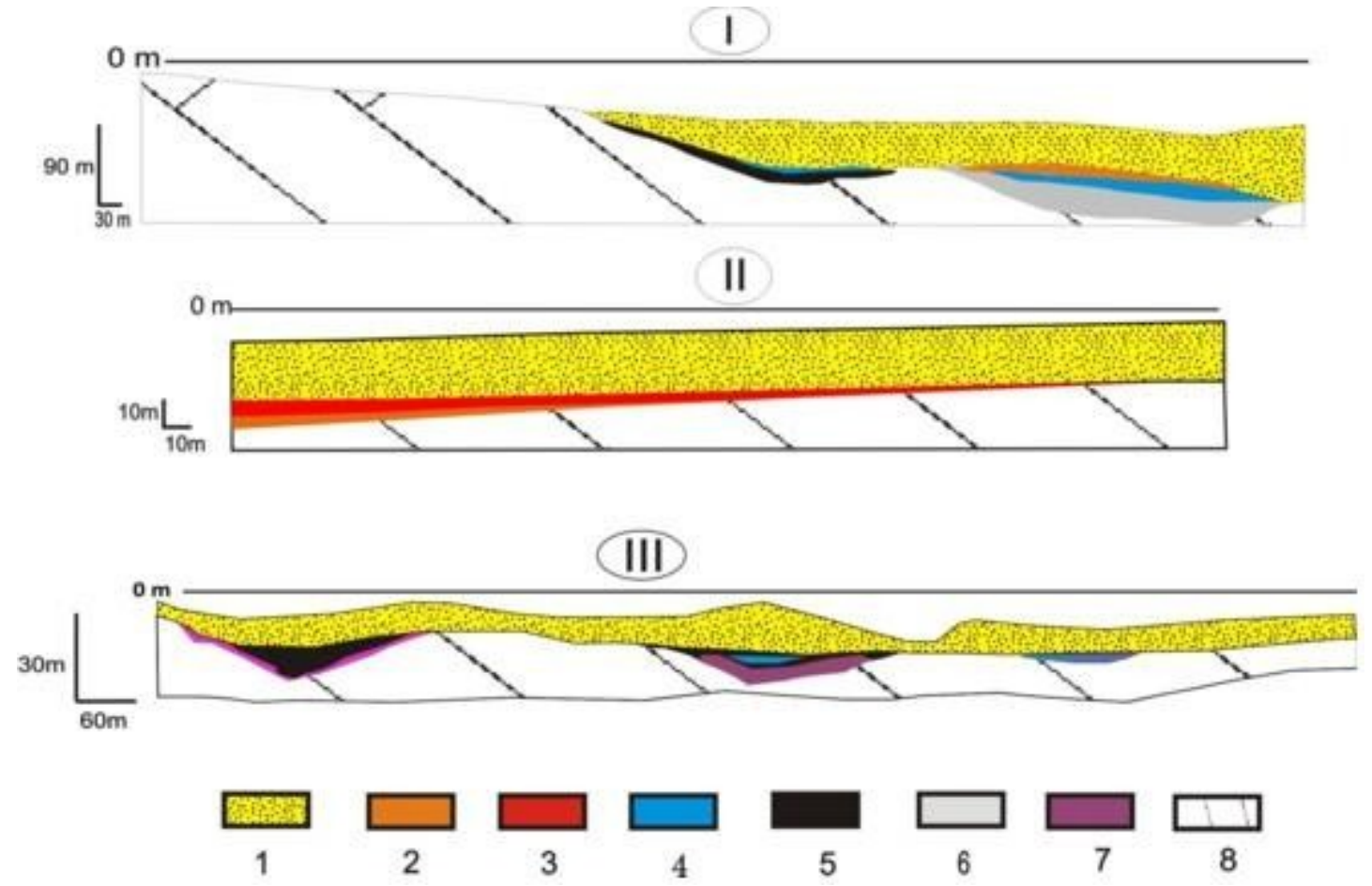

Figure 4. Coupes géologique à l'emprise des ouvrages du port Tanger Med (I)Digue principale, (II) Digue secondaire, (III) Quai à conteneurs

Légende : 1 Sables, 2 Sables silteux, 3 Argile sableuse, 4 Alluvions, 5 Silts sableux, 6 Substratum altéré, 7 Marne, 8 Substratum Grés-Pélites

\section{Analyse du risque de liquéfaction}

\subsection{Etude de l'aléa sismique}

L’étude de l'aléa sismique du Port Tanger Méditerranée est conduite d'abord selon une approche déterministe (CHERKAOUI, 2003) puis selon une approche probabiliste réalisée par MARTIN et al. (2004a ; 2004b) du bureau d'études GEOTER international. La première approche, contractuelle, est réalisée selon les pratiques françaises pour les centrales nucléaires et pour les sites d’installations classées pour la protection de l'environnement. Elle permet de définir les séismes de dimensionnement. La seconde, conforme aux principes de l'Eurocode 8, vise cependant à calculer les accélérations maximales du sol à différentes périodes de retour et de caler les niveaux de dimensionnement par rapport à ceux correspondant à ces périodes de retour.

Cette analyse du risque sismique a permis de retenir deux séismes de projet : un pour la source proche et un pour la source lointaine, correspondant aux séismes majorés de sécurité SMS de l'étude déterministe. Leurs caractéristiques de magnitude et d'accélération sont respectivement 4,7 et 0,24 $\mathrm{g}$ correspondant à une période de retour de 1975 ans pour la source proche et 8,5 et 0,093 $g$ correspondant à une période de 
retour inférieure à 475 ans pour la source lointaine. L'étude de la liquéfaction a été menée sur la base de ces résultats.

\subsection{Les essais in situ}

L'analyse du risque est conduite à partir des essais de pénétration statique CPTU ( $U$, pression interstitielle). Ces essais permettent de mesurer la pression interstitielle dans le sol en plus de la résistance à la pénétration du cône et du frottement latéral local sur le manchon situé immédiatement au-dessus de celui-ci.

\subsection{L’analyse du risque de liquéfaction et le calcul des facteurs de sécurité}

En l'absence de réglementation marocaine en la matière, cette analyse est réalisée selon les règles PS 92 en suivant la méthode empirique de YOUD et IDRISS (2001) qui comprend les phases suivantes :

Phase 1 : Vérification à partir d'essais en laboratoire, réalisés à partir des prélèvements issus de sondages carottés ou d'essais SPT, si les sols en place sont potentiellement liquéfiables sous sollicitations sismiques. Selon la norme NF P.06-13 sont considérés comme susceptibles de liquéfaction les sols sableux et les sols silteux présentant les caractéristiques suivantes :

- degré de saturation voisin de $100 \%$;

- granulométrie assez fortement uniforme: $D_{60} / D_{10}<15$;

- diamètre à D50 compris entre : 0.05mm et1.5mm ;

- contrainte verticale effective inférieure à 0.3MPa.

Les sols argileux présentant les caractéristiques suivantes :

- degré de saturation : $\mathrm{Sr}=100 \%$;

- diamètre à 15\% : $D_{15}$ supérieur à $5 \mu \mathrm{m}$;

- Limite de liquidité $W_{L}$ inférieure à $35 \%$;

- Teneur en eau W supérieure à 0,9 $W_{L}$.

Dans le Port Tanger Méditerranée, les sols remplissant strictement ces critères se situent dans :

- la frange supérieure du sondage SC 104 (0-4m) et vers 12,50 m de profondeur ;

- vers 10 à 13 m puis à 18 m de profondeur dans le sondage SC 105 ;

- de 0 à 12 m dans le sondage SC 106 ;

- de 7 à 12 m de profondeur dans le sondage SC 108.

Phase 2: si les formations sont potentiellement liquéfiables, on procède à une étude préliminaire du risque de liquéfaction en champ libre, en négligeant dans un premier temps l'effet de la structure sur le terrain. L'influence de l'interaction sol-structure sur le risque de liquéfaction est estimée en calculant l'influence favorable d'un chargement apporté par l'ouvrage au terrain.

L'évaluation du risque de liquéfaction est fondée sur le calcul d'un facteur de sécurité FS qui est défini comme le rapport entre la résistance cyclique normalisée du matériau 
$(C R R)$, et la sollicitation cyclique normalisée (CSR) induite par le séisme à la même profondeur.

$F S=C R R / C S R$

Le rapport $C S R$ en champ libre est déduit de l'accélération maximale du séisme à la surface du sol, $a_{\max }$, selon la formule suivante :

CSR $=0,65\left(a_{\max } / g\right)\left(\sigma_{v o} / \sigma_{v o}^{\prime}\right) r_{d}$

avec :

$a_{\max }$ : accélération maximale du séisme à la surface du sol,

$g$ : accélération de la pesanteur, $\left(9,81 \mathrm{~m} / \mathrm{s}^{2}\right)$,

$\sigma_{v o}$ : contrainte totale verticale,

$\sigma_{v o}^{\prime}$ : contrainte effective verticale,

$r_{d}$ : coefficient réducteur de la contrainte en fonction de la profondeur.

Pour l'étude de la liquéfaction, les paramètres du CPTu doivent être normalisés (ROBERTSON \& WRIDE, 1998). En effet, la résistance totale du cône qt et le frottement latéral $\mathrm{fs}_{\mathrm{s}}$ sont tout d'abord corrigés par la contrainte effective pour calculer $Q_{t}$ et $F r$ qui servent à l'établissement de la charte des types de comportements des sols (SBT) dont les différentes zones peuvent être représentées par l'indice Ic (ROBERTSON, 2010).

$Q_{t}=\left(q t-\sigma_{v o}\right) / \sigma_{v o}^{\prime}$

$F r=\left(f s /\left(q t-\sigma_{v o}\right)\right) * 100 \%$

$I_{c}=\left((3.47-\log Q)^{2}+(1.22+\log F)^{2}\right)^{1 / 2}$.

Ensuite qt est normalisée en introduisant les contraintes totales et effectives ( $\sigma_{v o}$ et $\sigma_{v_{v o}}$ ), la pression atmosphérique ( $\mathrm{Pa}$ ) et le facteur de normalisation n qui est fonction de Ic.

Cette normalisation se fait selon la formule suivante :

$Q_{t n}=\left(\left(q t-\sigma_{v o}^{\prime}\right) / P a\right)\left(P a / \sigma_{v o}^{\prime}\right)^{n}$

où $n=0.381\left(I_{c}\right)+0.05\left(\sigma_{v o}^{\prime} / p a\right)-0.15$.

Pour les sables silteux un autre coefficient correcteur Kc est introduit :

$Q t c n=K_{c} \times Q_{t n}$

$K_{c}=1$, si $I_{c}$ est inférieur ou égal à 1.64

$K_{c}=5.581 I_{c}{ }^{3}-0.403 I_{c}{ }^{4}-21.63 I c^{2}+33.75 I_{c}-17.88$, si Ic est compris entre 1.64 et 2.50

$K_{c}=610^{-7}(I c)^{16.76}$, si Ic est compris entre 2.50 et 2.70

La valeur de la résistance cyclique $C R R$ est calculée à partir de $Q t n$ si Ic est inférieur à 2.70 et à partir de Qtcn si Ic est supérieur à 2.70. Dans la pratique la $C R R$ est toujours calculée pour un séisme de référence de magnitude 7,5 , d'où la notation $C R R_{7,5}$. Pour ajuster cette valeur à des magnitudes différentes, plus fiables et plus fortes selon le cas d'étude, les auteurs (YOUD \& IDRISS, 2001) ont introduit un facteur pondérateur de la magnitude $M S F$, d'où la formule suivante :

$C R R=C R R_{7,5} \times M S F$

Le facteur MSF est calculé à partir d'un abaque en fonction de la magnitude. 
Le risque de liquéfaction est considéré nul lorsque le coefficient de sécurité $F S$ est supérieur à 2, peu probable pour une valeur de FS comprise entre 1.33 et 2 et probable pour des valeurs comprises entre 1 et 1.33. Pour des valeurs de FS inférieures à 1 le risque est quasi certain.

\section{Résultats de l'évaluation du risque de liquéfaction}

L’évaluation du risque de liquéfaction intéresse particulièrement l'emprise des ouvrages de protection où d'épaisses formations de sables et de sables silteux, très sensibles à ce phénomène, ont été repérées. Sous le quai à conteneur ces matériaux ont été dragués et substitués pour palier aux tassements inacceptables. Le calcul de FS au droit de la digue principale montre que celui-ci est inférieur à 1,3 dans les niveaux figurant au tableau 2 . Un exemple au niveau de l'essai CPT 25 dont les résultats sont donnés dans ce tableau, est montré sur la figure 5 .

Dans l'emprise de la digue secondaire comprenant les essais CPT32, СРТ33 et CРT34, tous les coefficients sont supérieurs à 1,3 et par conséquent aucun niveau à risque n’a été repéré voir figure 6.

Tableau 2. Profondeur des niveaux à risque à l'emplacement de la digue principale.

\begin{tabular}{|c|c|c|c|c|c|c|c|c|c|}
\hline & \multicolumn{4}{|c|}{ Source proche de magnitude $=4,7$} & \multicolumn{5}{|c|}{ Source lointaine de magnitude $=8,5$} \\
\hline & СРТ21 & СРТ22 & СРТ24 & СРT25 & СРT21 & СРТ22 & СРТ24 & СРT25 & СРТ27 \\
\hline 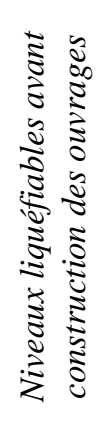 & $\begin{array}{l}6-6,6 \\
7,6-7,8\end{array}$ & $\begin{array}{l}0,4-6 \\
9,6-10 \\
12-12,2\end{array}$ & $\begin{array}{l}1,2-1,8 \\
2,2-2,4 \\
3,2-5\end{array}$ & $0,6-2,8$ & $\begin{array}{l}0,4-0,8 \\
4-4,2 \\
5,2-5,4 \\
5,8-6,8 \\
7,2-8,8\end{array}$ & $\mid \begin{array}{l}0,4-6,2 \\
8,4-8,8 \\
9,6-10,4 \\
12-12,4\end{array}$ & $\begin{array}{l}0,6-0,8 \\
1,2-2,4 \\
3,2-5,2\end{array}$ & $\mid \begin{array}{l}0,6-2,8 \\
6,2-6,6 \\
13,8-14 \\
15,4-16,2 \\
16,6-16,8 \\
17,2-17,4 \\
18,6-19\end{array}$ & $\begin{array}{l}18,6-19,2 \\
21,4-21,8\end{array}$ \\
\hline 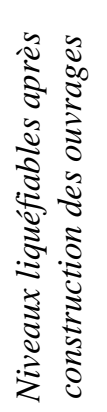 & Néant & $\begin{array}{l}0,8-3,6 \\
4,2-4,4\end{array}$ & $\begin{array}{l}3,2-4,2 \\
4,6-4,8\end{array}$ & $\begin{array}{l}0,6-0,8 \\
1,6-2,2\end{array}$ & $\begin{array}{l}6-6,6 \\
7,6-7,8\end{array}$ & $\mid \begin{array}{l}0,4-6 \\
8,6-8,8 \\
12-12,2\end{array}$ & $\begin{array}{l}1,2-2,4 \\
3,2-5\end{array}$ & $0,6-2,8$ & Néant \\
\hline
\end{tabular}




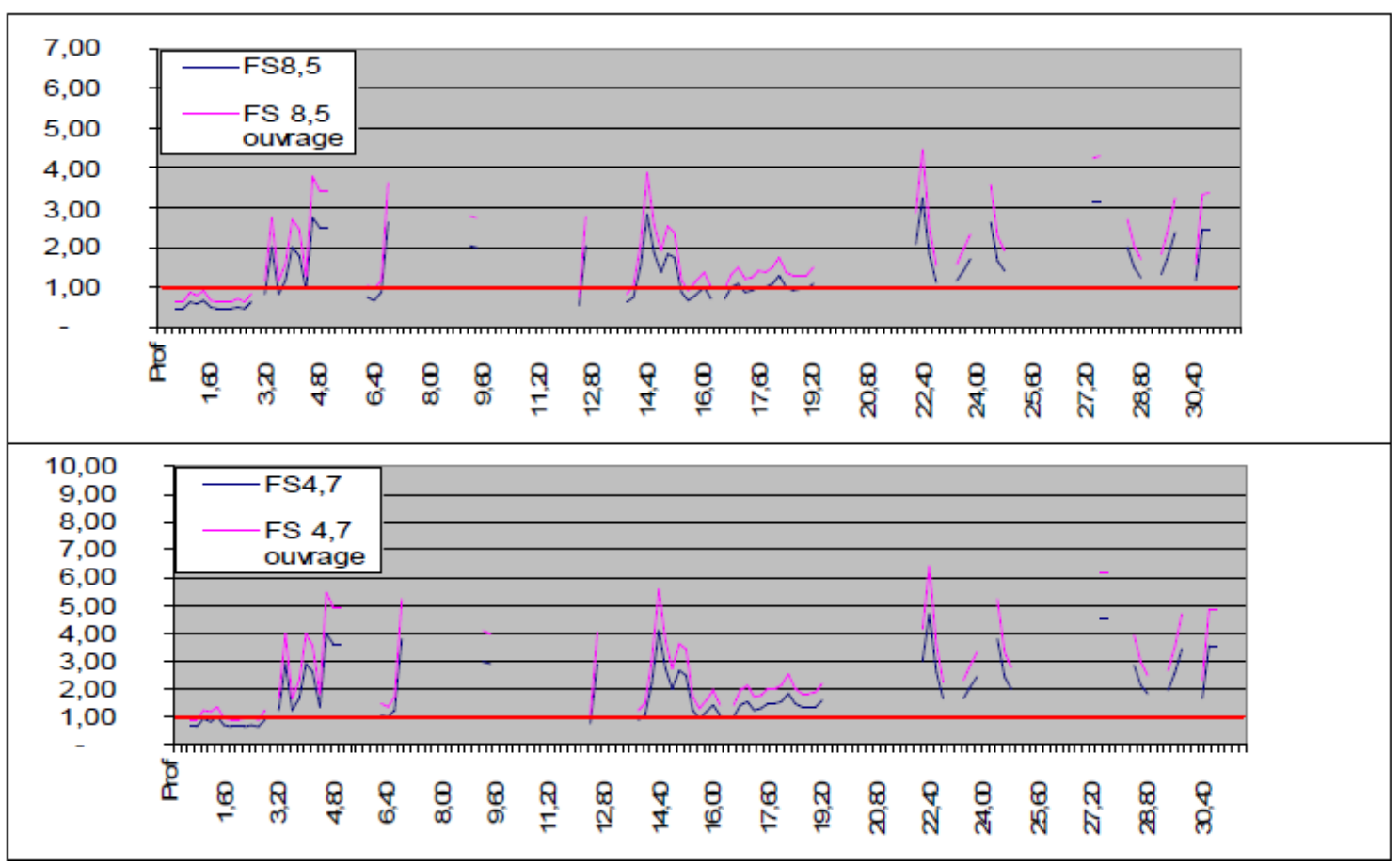

Figure 5. Analyse du risque de liquéfaction au niveau du CPT25 pour la digue principale (en abscisses la profondeur, en ordonnées le FS).

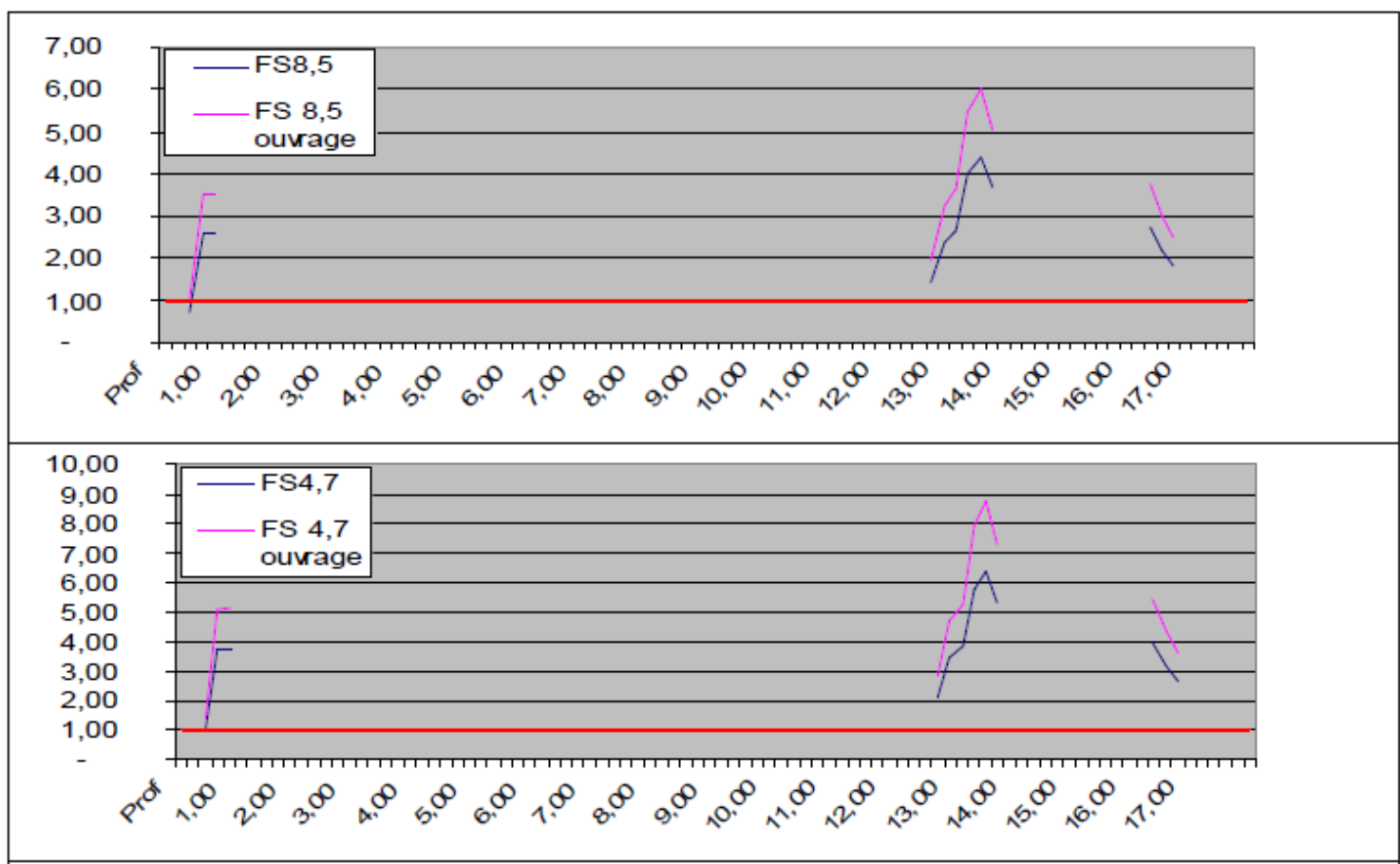

Figure 6. Analyse du risque de liquéfaction au niveau du CPT33 pour la digue secondaire (en abscisses la profondeur, en ordonnées le FS).

L’analyse des résultats a montré que le séisme lointain est plus favorable au phénomène de liquéfaction que le séisme de source proche. 
La charge de l'ouvrage atténue le potentiel de liquéfaction. Seuls subsistent quelques zones à risque comme dans la partie superficielle du sol (environ $3 \mathrm{~m}$ ) et quelques niveaux très réduits en épaisseur.

La zone critique coïncide avec les essais CPT22, CPT24 et CPT25 sur environ $460 \mathrm{~m}$ leur localisation est précisée sur la figure 7.

\section{Conclusion}

L'étude géologique et géotechnique du Port Tanger Mediterranée met en évidence l'existence de trois vallées dont l'ampleur est variable traversant le site du port et une dominance des sables et des sables silteux sous les ouvrages de protection susceptibles de liquéfier sous sollicitation sismique. L'analyse du risque, conduite sur la base d'essais CPTU et des niveaux des séismes retenus pour le projet, montre que la zone à risque se situe entre les CPT22 et CPT25. De plus, le séisme lointain est plus préjudiciable pour le terrain. La charge appliquée par l'ouvrage réduit ce potentiel de liquéfaction. Seule une zone à risque subsiste dans la partie superficielle du sol d'environ 3 m de hauteur.

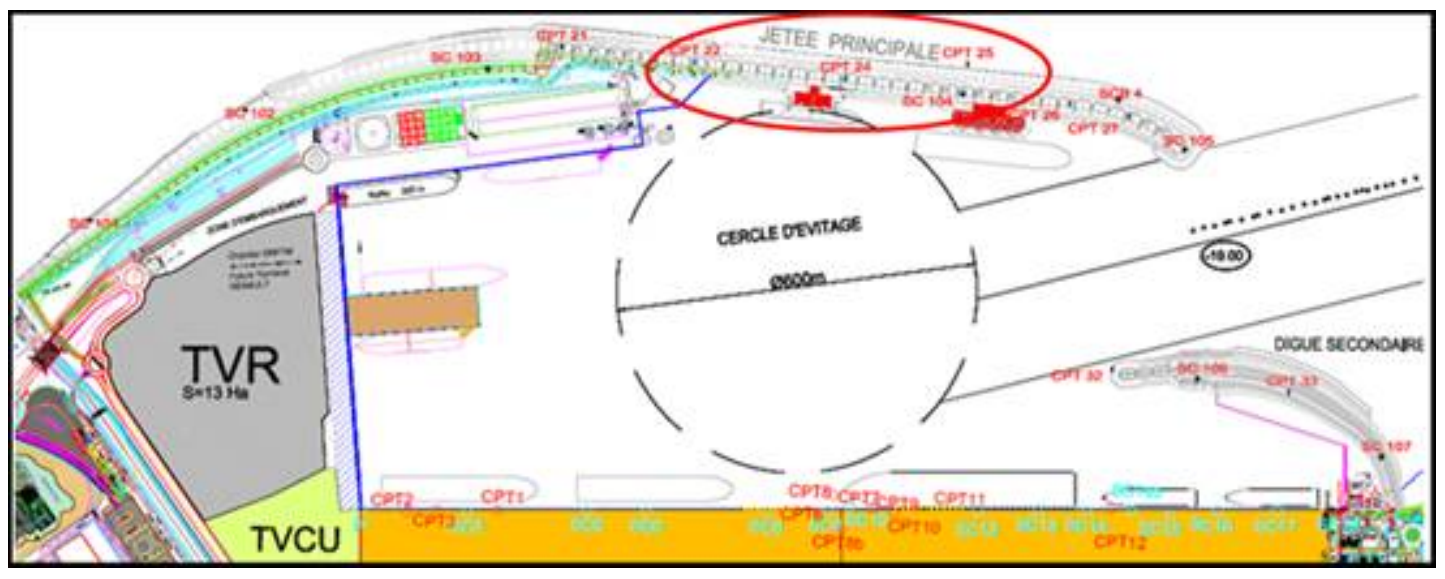

Figure 7. Localisation de la zone à risque de liquéfaction.

\section{Remerciement}

Ce travail a été réalisé avec l'aide de projets RS/2011/6 financé par le CNRST du Maroc.

\section{Références bibliographiques}

AOULAD MANSOUR M.R. (2007). Port Tanger Méditerranée : Etude géologique et géotechnique et évaluation du risque de liquéfaction. Rapport de projet de fin d'études, Ecole Nationale de l'Industrie Minérale, 92 p.

AOULAD MANSOUR M.R., MIHRAJE A., STITOU MESSARI J.E. (2011). Port Tanger Méditerranée: Etude géologique et géotechnique et analyse du risque de 
liquéfaction à partir des essais in situ. Conférence Méditerranéenne Côtière et Maritime Edition 2, Tanger, Maroc, pp 5-8. http://dx.doi.org/doi:10.5150/cmcm.2011.002

CHERKAOUI T.E. (2003). Evaluation de l'aléa sismique : port de TangerMéditerranée. Rapport d'étude.

MARTIN C., CARBON D., SECANELL R. (2004a). Evaluation probabiliste de l'aléa sismique sur le site du port de Tanger Méditerranée. GEOTER International Rapport GTI/TMSA/0105-50. 27 p.

MARTIN CH., CARBON D., SECANELL R. (2004b). Annexes -Evaluation probabiliste de l'aléa sismique sur le site du port de Tanger Méditerranée. GEOTER International Rapport GTI/TMSA/0105-50. 40 p.

ROBERTSON P.K., WRIDE C.E. (1998). Evaluating cyclic liquefaction potential using the cone penetration test. Canadian Geotechnical Journal, Ottawa, Vol. 35(3), pp 442459. http://dx.doi.org/10.1139/t98-017

ROBERTSON P.K. (2010). Evaluation of flow liquefaction and liquefied strength using the cone penetration test. Journal of Geotechnical and Geoenvironmental Engineering ASCE, Vol. 136 (6), pp 842-853. http://dx.doi.org/10.1061/(ASCE)GT.1943-5606.0000286

YOUD T.L., IDRISS I.M. (2001). Liquefaction resistance of soils: summary report from the 1996 NCEER and 1998 NCEER/NSF workshops on evaluation of liquefaction resistance of soils. Journal of Geotechnical and Geoenvironmental Engineering ASCE, Vol. 127 (4), pp 297-313. http://dx.doi.org/10.1061/(ASCE)1090-0241(2001)127:4(297) 